\title{
ON BESOV REGULARITY OF TEMPERATURES
}

\author{
HUGO AIMAR, IVANA GÓMEZ, AND BIBIANA IAFFEI
}

\begin{abstract}
We prove space-time parabolic Besov regularity in terms of integrability of Besov norms in the space variable for solutions of the heat equation on cylindrical regions based on Lipschitz domains.
\end{abstract}

\section{INTRODUCTION AND STATEMENT OF THE RESULT}

In [JK] Jerison and Kenig prove that, for harmonic functions on Lipschitz domain, some Besov norms are equivalent to Sobolev norms. On the other hand (see [DD]), estimates in Besov norms for solutions of elliptic problems on Lipschitz domains, become an important tool for the study of the rate of convergence for nonlinear approximation methods. In [AGI] the authors prove that for a temperature $u$, a weighted $L_{p}$ norm in space-time is bounded by a mixed norm of the type $L_{p}$ in time and Besov in space on a cylindrical domain with Lipschitz section.

In this note we investigate the simultaneous, in space and time, Besov regularity of solutions of the heat equation in terms of the $L_{p}$ integrability in time of space Besov regularity. A temperature on a cylindrical set $\Omega=D \times(0, T)$, with $D$ a domain on $\mathbb{R}^{d}$, is a solution of the heat equation $\frac{\partial u}{\partial t}=\Delta u$, where $\Delta$ is the Laplace operator on $D \subset \mathbb{R}^{d}$. The space of all temperatures $u$ in $\Omega$ shall be denoted by $\Theta(\Omega)$. Our main result is contained in the following statement.

Theorem 1.1. Let $D$ be a Lipschitz domain in $\mathbb{R}^{d}$ and $T>0$. Then

$$
\Theta(\Omega) \cap L_{p}\left((0, T) ; B_{p}^{\lambda}(D)\right) \subseteq \mathbb{B}_{p}^{\lambda-\varepsilon}(\Omega),
$$

for every $0<\lambda<1,1<p<\infty$ and every $0<\varepsilon<\lambda$.

Here $\mathbb{B}_{p}^{\alpha}(\Omega)$ is a parabolic Besov space which involves Besov regularity of order $\alpha$ in space variables and $\frac{\alpha}{2}$ in time. The precise definition that we shall give by interpolation, is contained in Section 2. There are some other approaches to anisotropic Besov spaces such us the one contained in [JM]. Even in general settings like spaces of homogeneous type, which contains the parabolic case, a theory of Besov regularity is quite developed, see for example [HS]. Let us point out that extensions of Theorem 1.1 for the case $\lambda \geq 1$ can be deduced from the action of derivatives on both scales of Besov spaces involved.

We would like to observe that this result can be seen as another manifestation of the regularizing property of diffusions. Of course without the restriction to the set of temperatures the inclusion of $L_{p}\left((0, T) ; B_{p}^{\lambda}(D)\right)$ in $\mathbb{B}_{p}^{\lambda-\varepsilon}(\Omega)$ is not true. In

2000 Mathematics Subject Classification. Primary 35B65, 35K05; Secondary 42B25.

Key words and phrases. Parabolic Besov Spaces, Heat Equation, Lipschitz Cylindrical Regions, Gradient Estimates. 
fact, for $\lambda$ large enough, and $\varepsilon$ small, the functions in $\mathbb{B}_{p}^{\lambda-\varepsilon}(\Omega)$ are continuous in $\Omega$.

Section 2 is devoted to briefly introduce the spaces of regularity considered here. Section 3 contains localizations of the main result in [AGI] which provide $L_{p}(\Omega)$ estimates for gradients of temperatures in terms of mixed Lebesgue-Besov norms in space-time. In Section 4 we give the proof of Theorem 1.1. The proof is, as in [JK], an application of the trace method of interpolation, taking care of the different types of points in the parabolic boundary of $\Omega$.

\section{Parabolic Besov spaces}

In the statement of Theorem 1.1 two classes of Besov spaces are involved. By $B_{p}^{\alpha}(D)$ we denote the standard elliptic Besov space $B_{p, p}^{\alpha}(D)$. By $\mathbb{B}_{p}^{\alpha}(\Omega)$ we denote a parabolic Besov space that we shall define precisely in this section.

The full scale of Besov spaces on the free space $\mathbb{R}^{d}$, usually denoted by $B_{p, q}^{\alpha}\left(\mathbb{R}^{d}\right)$, is well known and several equivalent characterizations are possible. Given an open subset $D$ in $\mathbb{R}^{d}$ one can define $B_{p, q}^{\alpha}(D)$ as the linear space of all the restrictions to $D$ of the functions in $B_{p, q}^{\alpha}\left(\mathbb{R}^{d}\right)$. We shall only deal with the case $p=q$ and for simplicity, we shall write $B_{p}^{\alpha}(D)$ instead of $B_{p, p}^{\alpha}(D)$. An intrinsic approach to $B_{p}^{\alpha}(D)$ is also possible. Under the assumption in Theorem 1.1, regarding the regularity of $D$, both approaches to $B_{p}^{\alpha}(D)$ are equivalent.

From the PDE's point of view, perhaps the most natural approach is the one provided by real interpolation between Lebesgue and Sobolev spaces. More precisely, we have that, for $0<\alpha<1$ and $1 \leq p \leq \infty$, the space $B_{p}^{\alpha}(D)$ coincides with the $\alpha$-interpolated between $L_{p}(D)$ and $W_{p}^{1}(D)$, usually denoted by $\left[L_{p}(D), W_{p}^{1}(D)\right]_{\alpha, p}$ (see $[\mathrm{BS}],[\mathrm{Pe}])$. When dealing with the parabolic spaces it will be convenient to realize that we can also get $B_{p}^{\alpha}(D)$ for $0<\alpha<1$ as $\frac{\alpha}{2}$-interpolated between $L_{p}(D)$ and $W_{p}^{2}(D)$. In other words

$$
B_{p}^{\alpha}(D)=\left[L_{p}(D), W_{p}^{2}(D)\right]_{\frac{\alpha}{2}, p}
$$

for $0<\alpha<1$.

Less standard are the parabolic Besov spaces. Even when a full scale of anisotropic Sobolev and Besov spaces can be considered we shall only introduce those which are strictly necessary for the proof our main result. As usual $\nabla$ denotes the gradient in space variables. By $\nabla^{2,1}$ we shall denote the vector valued differential operator which applied to a smooth function $v(x, t)$ defined on $\Omega$, produces the vector of all the second order spatial derivatives of $v$ and its first order derivative with respect to the time variable $t$. The anisotropic Sobolev space $W_{p}^{2,1}(\Omega)$ is the closure of $\mathscr{C}^{\infty}(\Omega)$ with respect to the norm

$$
\|v\|_{W_{p}^{2,1}(\Omega)}=\|v\|_{L_{p}(\Omega)}+\sum_{i=1}^{d}\left\|\frac{\partial v}{\partial x_{i}}\right\|_{L_{p}(\Omega)}+\sum_{i=1}^{d} \sum_{j=1}^{d}\left\|\frac{\partial^{2} v}{\partial x_{i} \partial x_{j}}\right\|_{L_{p}(\Omega)}+\left\|\frac{\partial v}{\partial t}\right\|_{L_{p}(\Omega)} .
$$

With this notation in mind for $W_{p}^{2,1}(\Omega)$, we may think of $L_{p}(\Omega)$ as $W_{p}^{0,0}(\Omega)$, because no regularity in any variable is required. Hence we can look for the interpolated spaces between $W_{p}^{0,0}(\Omega)$ and $W_{p}^{2,1}(\Omega)$. For $0<\alpha<2$ we define

$$
B_{p}^{\alpha, \frac{\alpha}{2}}(\Omega)=\left[L_{p}(\Omega), W_{p}^{2,1}(\Omega)\right]_{\frac{\alpha}{2}, p} .
$$


For simplicity we introduce the notation $\mathbb{B}_{p}^{\alpha}(\Omega)$ for the space $B_{p}^{\alpha, \frac{\alpha}{2}}(\Omega)$. Notice that the intersection of $\mathbb{B}_{p}^{\alpha}(\Omega)=B_{p}^{\alpha, \frac{\alpha}{2}}(\Omega)$ with the space of functions which do not depend on time is, from $(2.1)$, precisely $B_{p}^{\alpha}(D)$. On the other hand the intersection of $\mathbb{B}_{p}^{\alpha}(\Omega)$ with the space of functions on $\Omega$ which do not depend on the space variable, is the space $B_{p}^{\frac{\alpha}{2}}((0, T))$.

The only subtle point in the proof of our main result is contained in the so called trace space method. This approach was already used by Jerison and Kenig in the elliptic case in order to show that for harmonic functions, Besov regularity is equivalent to Sobolev regularity. For the sake of completeness we shall briefly describe the method for the particular case of parabolic spaces. We shall follow, with our special function spaces, the scheme introduced in page 72 of the book Interpolation Spaces by Bergh and Löfström (see [BL]).

Since we have to deal with the problem of proving that a temperature belongs to a particular parabolic Besov space, we only state the sufficient conditions contained in Corollary 3.12.3 in [BL] adapted to our particular situation.

Let $A_{0}=W_{p}^{2,1}(\Omega)$ and $A_{1}=L_{p}(\Omega)$. If we want to show that a function $v=$ $v(x, t)$ defined on $\Omega$ belongs to the space

$$
\mathbb{B}_{p}^{\alpha}(\Omega)=\left[L_{p}(\Omega), W_{p}^{2,1}(\Omega)\right]_{\frac{\alpha}{2}, p}=\left[W_{p}^{2,1}(\Omega), L_{p}(\Omega)\right]_{1-\frac{\alpha}{2}, p}=\left[A_{0}, A_{1}\right]_{1-\frac{\alpha}{2}, p}
$$

we only have to find an $m$-times differentiable function $f: \mathbb{R}^{+} \rightarrow W_{p}^{2,1}(\Omega)+L_{p}(\Omega)$ with $f(0)=v$ (in the sense that $f(s) \rightarrow v$ in $W_{p}^{2,1}(\Omega)+L_{p}(\Omega)$ as $\left.s \rightarrow 0\right)$, two positive numbers $\eta_{0}$ and $\eta_{1}$ with $\eta_{1}<m$ such that $1-\frac{\alpha}{2}=\frac{\eta_{0}}{\eta_{0}+m-\eta_{1}}$ and the integrals

$$
\int_{0}^{\infty}\left\|s^{\eta_{0}} f(s)\right\|_{W_{p}^{2,1}(\Omega)}^{p} \frac{d s}{s}
$$

and

$$
\int_{0}^{\infty}\left\|s^{\eta_{1}} f^{(m)}(s)\right\|_{L_{p}(\Omega)}^{p} \frac{d s}{s}
$$

are both finite. For our particular problem we shall use only two realizations of the above described situation. Precisely

(2.a) $v \in \mathbb{B}_{p}^{\alpha}(\Omega)$ if there exist $f: \mathbb{R}^{+} \rightarrow W_{p}^{2,1}(\Omega)+L_{p}(\Omega)$ with $f(0)=v$ and $\eta_{0}>0$ such that

$$
\int_{0}^{\infty}\left\|s^{\eta_{0}} f(s)\right\|_{W_{p}^{2,1}(\Omega)}^{p} \frac{d s}{s}+\int_{0}^{\infty}\left\|s^{2-\frac{\alpha}{2-\alpha} \eta_{0}} f^{\prime \prime}(s)\right\|_{L_{p}(\Omega)}^{p} \frac{d s}{s}<\infty ;
$$

(2.b) $v \in \mathbb{B}_{p}^{\alpha}(\Omega)$ if there exist $g: \mathbb{R}^{+} \rightarrow W_{p}^{2,1}(\Omega)+L_{p}(\Omega)$ with $g(0)=v$ and $\tau_{0}>0$ such that

$$
\int_{0}^{\infty}\left\|s^{\tau_{0}} g(s)\right\|_{W_{p}^{2,1}(\Omega)}^{p} \frac{d s}{s}+\int_{0}^{\infty}\left\|s^{1-\frac{\alpha}{2-\alpha} \tau_{0}} g^{\prime}(s)\right\|_{L_{p}(\Omega)}^{p} \frac{d s}{s}<\infty .
$$

\section{Gradient estimates And localizations of temperatures}

In this note $D$ is a Lipschitz domain in $\mathbb{R}^{d}$. Let us start by stating a local version in time of Corollary 6.2 in [AGI]. Given a bounded Lipschitz domain $D$ in $\mathbb{R}^{d}$ and $T>0$ we shall write $\delta=\delta(x ; t)$ to denote the parabolic distance of $(x ; t) \in \Omega=$ 
$D \times(0, T)$ to the parabolic boundary of $\Omega, \partial_{\text {par }} \Omega=(\partial D \times[0, T)) \cup(D \times\{0\})$. In other words with $\rho((x ; t),(y ; s))=\max \{|x-y|, \sqrt{|t-s|}\}$

$$
\delta(x ; t)=\inf \left\{\rho((x ; t),(y ; s)):(y ; s) \in \partial_{\operatorname{par}} \Omega\right\} .
$$

Theorem 3.1. Let $0<\lambda<1$ and $1<p \leq \infty$ be given. There exists a constant $C$ depending on $p, \lambda$ and $D$ but not on $T$ such that for every temperature $u$ in $\Omega$ we have the inequalities

$$
\begin{gathered}
\left\|\delta^{1-\lambda}|\nabla u|\right\|_{L_{p}(\Omega)} \leq C\|u\|_{L_{p}\left((0, T) ; B_{p}^{\lambda}(D)\right)}, \\
\left\|\delta^{2-\lambda}\left|\nabla^{2,1} u\right|\right\|_{L_{p}(\Omega)} \leq C\|u\|_{L_{p}\left((0, T) ; B_{p}^{\lambda}(D)\right)} .
\end{gathered}
$$

The proof is the same that the proof of Corollary 6.2 in [AGI] once a pointwise maximal estimate of the type

$$
\sup _{0<\delta<\delta(x ; t)} \delta^{1-\lambda}\left|\nabla\left(K_{\delta} * u\right)(x ; t)\right| \leq C M_{T}^{-}\left[M_{D}^{\#, \lambda} u\right](x ; t),
$$

is proved. Here $K_{\delta}(x ; t)=\frac{1}{\delta^{d+2}} K\left(\frac{x}{\delta} ; \frac{t}{\delta^{2}}\right)$ with $K(x ; t)=\frac{1}{4} \frac{|x|^{2}}{t^{2}} \eta\left((4 \pi t)^{\frac{1}{2}} e^{\frac{|x|^{2}}{d 4 t}}\right)$ and $\eta$ is a nonnegative $\mathscr{C}^{\infty}(\mathbb{R})$ function defined on $\mathbb{R}$ supported in $[0,1]$ satisfying $\int_{0}^{1} \eta(r) r^{d-1} d r=d^{-1}$. On the right hand side of the above inequality $M_{T}^{-}$is the onesided, one dimensional Hardy-Littlewood maximal operator on the interval $[0, T]$, in other words for $t \in[0, T]$

$$
M_{T}^{-} g(t)=\sup _{0<h<t} \frac{1}{h} \int_{t-h}^{t}|g(s)| d s .
$$

On the other hand $M_{D}^{\#, \lambda}$ is the Calderón sharp maximal operator for the space variable on $D$, precisely

$$
M_{D}^{\#, \lambda} f(x)=\sup _{0<\delta<d(x)} \frac{1}{|B(x, \delta)|^{1+\frac{\lambda}{d}}} \int_{B(x, \delta)}|f(y)-f(x)| d y,
$$

where $d(x)=\inf \{|x-y|: y \in \partial D\}$. We point out that (3.3) follows from the mean value formula for solutions of the heat equation as in the proof of Theorem 5.1 in [AGI].

The fact that $u$ is a temperature in Theorem 3.1 is used in the required mean value representation and in order to obtain estimates for the partial derivative with respect to time in terms of second order space derivatives. The next result, which shall be used in $\S 4$, proves that even when a smooth localization of a temperature is not a temperature, estimates like those in (3.1) and (3.2) are still true for such functions.

Lemma 3.2. Let $u$ be a temperature in $\Omega$. Let $\zeta \in \mathscr{C}^{\infty}\left(\mathbb{R}^{d+1}\right)$. Then the function $v=\zeta u$ defined in $\Omega$ satisfies the inequalities

$$
\begin{gathered}
\|v\|_{L_{p}(\Omega)} \leq C\|u\|_{L_{p}\left((0, T) ; B_{p}^{\lambda}(D)\right)} \\
\left\|\delta^{1-\lambda}|\nabla v|\right\|_{L_{p}(\Omega)} \leq C\|u\|_{L_{p}\left((0, T) ; B_{p}^{\lambda}(D)\right)} \\
\left\|\delta^{2-\lambda}\left|\nabla^{2,1} v\right|\right\|_{L_{p}(\Omega)} \leq C\|u\|_{L_{p}\left((0, T) ; B_{p}^{\lambda}(D)\right)}
\end{gathered}
$$

for some constant $C$ which is independent of $u$. 
Proof. Let us first notice that (3.4) follows from the boundedness of $\zeta$ on $\bar{\Omega}$ and from the inequality $\|u\|_{L_{p}\left((0, T) ; B_{p}^{\lambda}(D)\right)} \geq\|u\|_{L_{p}(\Omega)}$.

On the other hand, since also each derivative of $\zeta$ is bounded on $\bar{\Omega}$ and from Leibniz rule we have

$$
\begin{aligned}
\frac{\partial v}{\partial x_{i}} & =\zeta \frac{\partial u}{\partial x_{i}}+\frac{\partial \zeta}{\partial x_{i}} u \\
\frac{\partial^{2} v}{\partial x_{j} \partial x_{i}} & =\zeta \frac{\partial^{2} u}{\partial x_{j} \partial x_{i}}+2 \frac{\partial \zeta}{\partial x_{j}} \frac{\partial u}{\partial x_{i}}+\frac{\partial^{2} \zeta}{\partial x_{j} \partial x_{i}} u \\
\frac{\partial v}{\partial t} & =\zeta \frac{\partial u}{\partial t}+\frac{\partial \zeta}{\partial t} u
\end{aligned}
$$

in order to prove (3.5) and (3.6) it is enough to estimate the $L_{p}(\Omega)$ norms of the functions $\delta^{1-\lambda} u, \delta^{2-\lambda} u, \delta^{2-\lambda}|\nabla u|$ by $\|u\|_{L_{p}\left((0, T) ; B_{p}^{\lambda}(D)\right)}$. Since $D$ is bounded we have that $\left|\delta^{1-\lambda} u\right| \leq C|u|$ and $\left|\delta^{2-\lambda} u\right| \leq C|u|$. On the other hand, $\left|\delta^{2-\lambda} \nabla u\right| \leq$ $C\left|\delta^{1-\lambda} \nabla u\right|$ and the result follows from (3.1).

What is left of this section is devoted to produce a smooth partition of the identity in $\bar{\Omega}$. We shall closely, and as a for as possible, follow the elliptic notation introduced in [JK]. Precisely, the set $D$ is a bounded and open set in $\mathbb{R}^{d}$ for which there exists a positive number $r$ small enough such that for each point $x_{0} \in \partial D$ the set $D \cap B\left(x_{0}, r\right)$ is the set above the graph of a Lipschitz function $\varphi$ of $d-1$ variables $x^{\prime}$ in some local orthogonal coordinate system $\left(x^{\prime}, y\right)$ around $x_{0}$. Since $\partial D$ is compact there exists a constant $M$ independent of $x_{0} \in \partial D$ such that $|\nabla \varphi| \leq M$.

This localization at the boundary of the elliptic domain $D$, induces a classification of the points in $\Omega=D \times(0, T)$ in four different types according to its relative position with respect to the parabolic boundary of $\Omega$. Precisely, with $r>0$ given by the Lipschitz character of $\partial D$, we shall write $\mathcal{O}_{\text {I }}$ to denote the set of points in $\Omega$ with parabolic distance to the parabolic boundary, $\partial_{\text {par }} \Omega$, of $\Omega$ larger than $\frac{r}{2}$. In other words,

$$
\mathcal{O}_{\mathrm{I}}=\left\{(x ; t) \in \Omega: \rho\left((x ; t) ; \partial_{\mathrm{par}} \Omega\right)>\frac{r}{2}\right\}=\left\{(x ; t) \in \Omega: d(x)>\frac{r}{2} \text { and } t>r^{2}\right\} .
$$

By $\mathcal{O}_{\text {II }}$ we shall denote any of the cylindrical domains of the form $B(x, r) \times\left(r^{2}, T\right)$ with $x \in \partial D$. Let

$$
\mathcal{O}_{\text {III }}=\left\{(x ; t) \in \mathbb{R}^{d+1}: x \in D, d(x)>\frac{r}{2} \text { and }|t|<2 r^{2}\right\} .
$$

Finally, $\mathcal{O}_{\mathrm{IV}}$ shall denote any of the cylindrical domains of the form $B(x, r) \times$ $\left(-2 r^{2}, 2 r^{2}\right)$ with $x \in \partial D$.

Since $\partial D$ is compact, the family of open sets containing $\mathcal{O}_{\text {I }}, \mathcal{O}_{\text {III }}$ and a finite number of sets of type $\mathcal{O}_{\text {II }}$ and type $\mathcal{O}_{\text {IV }}$ provides an open covering of the parabolic closure of $\Omega\left(\Omega \cup \partial_{\mathrm{par}} \Omega\right)$. To precise the notation let us say that the family

$\left\{\mathcal{O}_{\mathrm{I}}, \mathcal{O}_{\mathrm{III}}\right\} \cup\left\{B\left(x_{i}, r\right) \times\left(-2 r^{2}, 2 r^{2}\right): i=1, \ldots, i_{0}\right\} \cup\left\{B\left(x_{i}, r\right) \times\left(r^{2}, T\right): i=1, \ldots, i_{0}\right\}$

is a finite open covering of the parabolic closure of $\Omega, x_{i} \in \partial D$. Hence we can find a corresponding sequence $\zeta_{\mathrm{I}}, \zeta_{\mathrm{III}}, \zeta_{\mathrm{IV}}^{i}$ and $\zeta_{\mathrm{II}}^{i}, i=1, \ldots, i_{0}$, of $\mathscr{C}^{\infty}\left(\mathbb{R}^{d+1}\right)$ functions such that $\zeta_{\mathrm{I}}+\zeta_{\mathrm{II}}+\sum_{i=1}^{i_{0}}\left(\zeta_{\mathrm{II}}^{i}+\zeta_{\mathrm{IV}}^{i}\right) \equiv 1$ on the parabolic closure of $\Omega$. Moreover, $\zeta_{\text {III }}$ and each $\zeta_{\mathrm{IV}}^{i}$ are compactly supported on $\mathcal{O}_{\mathrm{III}}$ and $B\left(x_{i}, r\right) \times\left(-2 r^{2}, 2 r^{2}\right)$ respectively. The function $\zeta_{\mathrm{I}}$ instead, vanishes on the parabolic boundary of the cylindrical domain $\mathcal{O}_{\mathrm{I}}$. The same is true for each $\zeta_{\mathrm{II}}^{i}$, in other words, $\zeta_{\mathrm{II}}^{i} \equiv 0$ on 

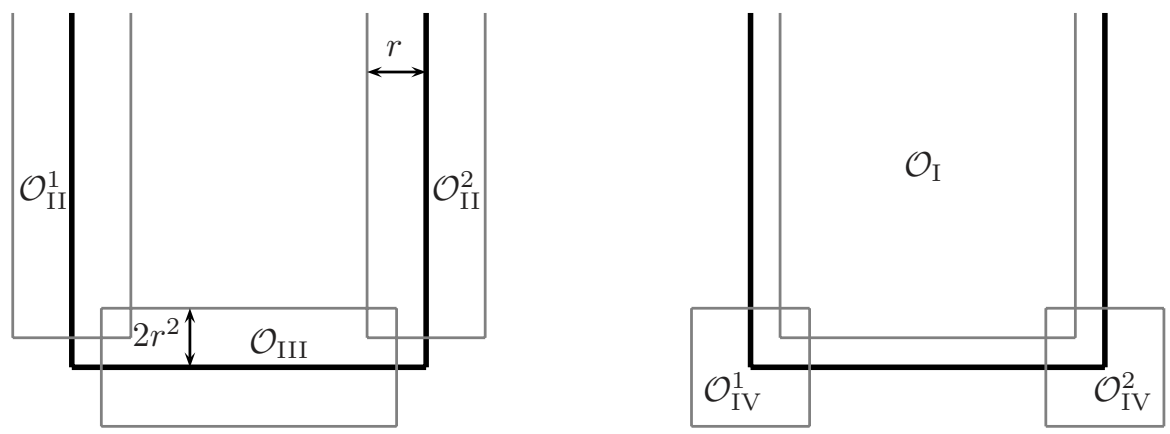

Figure 1. Decomposition for $d=1$

the parabolic boundary of the cylindrical domain $B\left(x_{i}, r\right) \times\left(r^{2}, T\right)$. We give an schematic representation of this decomposition for the simple case of $d=1$ and $D=(0,1)$ in Figure 1 .

\section{Proof of Theorem 1.1}

Take $u$ a temperature in $\Omega$ such that $u \in L_{p}\left((0, T) ; B_{p}^{\lambda}(D)\right)$. We have to check that $u$ belongs to $\mathbb{B}_{p}^{\lambda-\varepsilon}(\Omega)$ for every $0<\varepsilon<\lambda$. Notice first that since the number of functions $\zeta_{\mathrm{I}}, \zeta_{\mathrm{III}}, \zeta_{\mathrm{IV}}^{i}$ and $\zeta_{\mathrm{II}}^{i}$ is finite and depends only on $D$, and since

$$
\begin{aligned}
u & =u \zeta_{\mathrm{I}}+u \zeta_{\mathrm{III}}+\sum_{i=1}^{i_{0}}\left(u \zeta_{\mathrm{II}}^{i}+u \zeta_{\mathrm{IV}}^{i}\right) \\
& =v_{\mathrm{I}}+v_{\mathrm{III}}+\sum_{i=1}^{i_{0}}\left(v_{\mathrm{II}}^{i}+v_{\mathrm{IV}}^{i}\right)
\end{aligned}
$$

it will be enough to prove that each one of the terms in the sum on the right hand side above belongs to $\mathbb{B}_{p}^{\lambda-\varepsilon}(\Omega)$ for $0<\varepsilon<\lambda$.

We shall prove that each one of the four different types $v_{\mathrm{I}}, v_{\mathrm{III}}, v_{\mathrm{II}}^{i}$ and $v_{\mathrm{IV}}^{i}$ of localizations of $u$ belong to $\mathbb{B}_{p}^{\lambda-\varepsilon}(\Omega)$. For each such a type of localization we aim to prove the finiteness of the $\mathbb{B}_{p}^{\lambda-\varepsilon}(\Omega)$ norm from the fact that $u \in \Theta(\Omega) \cap$ $L_{p}\left((0, T) ; B_{p}^{\lambda}(D)\right)$. For $\zeta$ of type I, since $v=\zeta u$ vanishes on a neighborhood of the parabolic boundary of $\Omega$ and $u \in \Theta(\Omega)$, we have that $v$ belongs to any Besov space since it certainly belongs to $W_{p}^{2,1}(\Omega)$. For each one of the other three types (II, III and IV) of localization functions, we shall choose an adequate function $f$ (or $g$ ), and adequate number $\eta_{0}$ (or $\tau_{0}$ ) in (2.a) (or (2.b)) with $\alpha=\lambda-\varepsilon$ in order to prove the finiteness of $\mathbb{B}_{p}^{\lambda-\varepsilon}(\Omega)$.

EStimates FOR $v=v_{\mathrm{II}}^{i}$. In this case $v=v_{\mathrm{II}}^{i}=u \zeta_{\mathrm{II}}^{i}$. Assume that $\mathcal{O}_{\mathrm{II}}^{i}=$ $B\left(p_{i}, r\right) \times\left(r^{2}, T\right)$, with $p_{i} \in \partial D$. Since the Laplace operator is invariant under translations and under orthogonal transformations of $\mathbb{R}^{d}$, there is no loss of generality by assuming that $p_{i}=0$ and that the coordinate system $x=\left(x^{\prime}, y\right) ; x^{\prime} \in \mathbb{R}^{d-1}$, 
$y \in \mathbb{R}$ is the one for which locally, inside $B(0, r)$, the boundary of $D$ coincides with the graph of the Lipschitz function $\varphi$.

With the above convention we have that the function $v$ is given in this coordinate system by $v\left(x^{\prime}, y ; t\right)=\zeta\left(x^{\prime}, y ; t\right) u\left(x^{\prime}, y ; t\right)$ where $\zeta$ is of type II. Let $\theta(s)$ be a nonnegative $\mathscr{C}^{\infty}$ function on $\mathbb{R}$ supported in $\left(-\frac{r}{4}, \frac{r}{4}\right)$ and equals to one on the interval $\left[-\frac{r}{8}, \frac{r}{8}\right]$. In order to show that this function $v$ belongs to $\mathbb{B}_{p}^{\lambda-\varepsilon}(\Omega)$ we shall use (2.a) with $\eta_{0}=2-\lambda+\varepsilon(0<\varepsilon<\lambda), \alpha=\lambda-\varepsilon$ and $f(s)$ is the function on $\Omega$ which takes the value $v\left(x^{\prime}, y+s ; t\right) \theta(s)$ at the point $(x ; t)=\left(x^{\prime}, y ; t\right) \in \Omega$. In other words $f(s)(x ; t)=v\left(x^{\prime}, y+s ; t\right) \theta(s)$. Notice that the required condition $f(0)=v$ clearly holds from the choice of $f(s)$ which actually is the natural extension of the $f$ used in $[\mathrm{JK}]$ for the elliptic case. In the proof of (2.2) we shall also be proving that $f$ takes values in the space $W_{p}^{2,1}(\Omega)+L_{p}(\Omega)$.

Let us start by proving the finiteness of the first term on the left hand side of (2.2). For fixed $s>0$ and $T>t>0$ we shall also use the expression $f(s, t)$ to denote the function defined on $D$ which takes the value $f(s)(x ; t)$ at each point $x \in D$. Hence we shall start estimating for $t$ and $s$ fixed the $L_{p}(D)$ norms of the functions $f(s, t), \nabla f(s, t)$ and $\nabla^{2,1} f(s, t)$.

Since

$$
\begin{aligned}
\|f(s, t)\|_{L_{p}(D)}^{p} & =\iint_{D}\left|v\left(x^{\prime}, y+s ; t\right)\right|^{p} \theta^{p}(s) d x^{\prime} d y \\
& =\theta^{p}(s) \iint_{D}\left|v\left(x^{\prime}, y+s ; t\right)\right|^{p} d x^{\prime} d y \\
& =\theta^{p}(s) \int_{x^{\prime} \in B_{d-1}(0, r)}\left(\int_{\varphi\left(x^{\prime}\right)}^{+\infty}\left|v\left(x^{\prime}, y+s ; t\right)\right|^{p} d y\right) d x^{\prime} \\
& =\theta^{p}(s) \int_{x^{\prime} \in B_{d-1}(0, r)}\left(\int_{\varphi\left(x^{\prime}\right)+s}^{+\infty}\left|v\left(x^{\prime}, y ; t\right)\right|^{p} d y\right) d x^{\prime} \\
& \leq \theta^{p}(s) \int_{x^{\prime} \in B_{d-1}(0, r)}\left(\int_{\varphi\left(x^{\prime}\right)}^{+\infty}\left|v\left(x^{\prime}, y ; t\right)\right|^{p} d y\right) d x^{\prime} \\
& =\theta^{p}(s) \iint_{D}|v|^{p} d x,
\end{aligned}
$$

by integration in the variable $t$, we get from (3.4) in Lemma 3.2 that

$$
\|f(s)\|_{L_{p}(\Omega)}^{p} \leq C \theta^{p}(s)\|u\|_{L_{p}\left((0, T) ; B_{p}^{\lambda}(D)\right)}^{p} .
$$

For $\nabla f(s, t)$ if $x_{i}, i=1, \ldots, d-1$, is any one of the $d-1$ components of $x^{\prime}$ and $x_{d}=y$, we have that

$$
\begin{aligned}
\left\|\frac{\partial f}{\partial x_{i}}(s, t)\right\|_{L_{p}(D)}^{p} & =\iint_{D}\left|\frac{\partial v}{\partial x_{i}}\left(x^{\prime}, y+s ; t\right) \theta(s)\right|^{p} d x^{\prime} d y \\
& =\frac{\theta^{p}(s)}{s^{(1-\lambda) p}} \iint_{D}\left|s^{1-\lambda} \frac{\partial v}{\partial x_{i}}\left(x^{\prime}, y+s ; t\right)\right|^{p} d x^{\prime} d y .
\end{aligned}
$$

Now, we claim that $c s \leq d\left(x^{\prime}, y+s\right)$ for $x=\left(x^{\prime}, y\right) \in D$, where $d(z)$ is the distance of the point $z \in D$ to the boundary $\partial D$ of $D$, and $c$ is some geometric constant. On the other hand since the truncation function $\zeta$ is of type II, then for those values of 
$t$ for which $\zeta$ and $v$ do not vanish we have that $d\left(x^{\prime}, y+s\right)=\delta\left(x^{\prime}, y+s ; t\right)$. Hence

$$
\begin{aligned}
\left\|\frac{\partial f}{\partial x_{i}}(s, t)\right\|_{L_{p}(D)}^{p} & \leq c \frac{\theta^{p}(s)}{s^{(1-\lambda) p}} \iint_{D}\left|\delta^{1-\lambda}\left(x^{\prime}, y+s ; t\right) \frac{\partial v}{\partial x_{i}}\left(x^{\prime}, y+s ; t\right)\right|^{p} d x^{\prime} d y \\
& \leq c \frac{\theta^{p}(s)}{s^{(1-\lambda) p}} \iint_{D}\left|\delta^{1-\lambda} \frac{\partial v}{\partial x_{i}}\right|^{p} d x
\end{aligned}
$$

for $i=1, \ldots, d, s>0$ and $0<t<T$. In the last inequality an argument similar to the one used in the proof of (4.1) can be used. After integration in the variable $t$ and application of (3.5) we get

$$
\||\nabla f(s)|\|_{L_{p}(\Omega)}^{p} \leq C \frac{\theta^{p}(s)}{s^{(1-\lambda) p}}\|u\|_{L_{p}\left((0, T) ; B_{p}^{\lambda}(D)\right)}^{p} \cdot
$$

For $\nabla^{2,1} f(s, t)$, the second order space derivatives can be bounded with the same geometric argument in the following way

$$
\begin{aligned}
\left\|\frac{\partial^{2} f}{\partial x_{j} \partial x_{i}}(s, t)\right\|_{L_{p}(D)}^{p} & =\iint_{D}\left|\frac{\partial^{2} v}{\partial x_{j} \partial x_{i}}\left(x^{\prime}, y+s ; t\right) \theta(s)\right|^{p} d x^{\prime} d y \\
& =\frac{\theta^{p}(s)}{s^{(2-\lambda) p}} \iint_{D}\left|s^{2-\lambda} \frac{\partial^{2} v}{\partial x_{j} \partial x_{i}}\left(x^{\prime}, y+s ; t\right)\right|^{p} d x^{\prime} d y \\
& \leq c \frac{\theta^{p}(s)}{s^{(2-\lambda) p}} \iint_{D}\left|\delta^{2-\lambda}\left(x^{\prime}, y+s ; t\right) \frac{\partial^{2} v}{\partial x_{j} \partial x_{i}}\left(x^{\prime}, y+s ; t\right)\right|^{p} d x^{\prime} d y \\
& \leq c \frac{\theta^{p}(s)}{s^{(2-\lambda) p}} \iint_{D}\left|\delta^{2-\lambda} \frac{\partial^{2} v}{\partial x_{j} \partial x_{i}}\right|^{p} d x .
\end{aligned}
$$

For $\frac{\partial f}{\partial t}$ a similar argument gives

$$
\left\|\frac{\partial f}{\partial t}(s, t)\right\|_{L_{p}(D)}^{p} \leq c \frac{\theta^{p}(s)}{s^{(2-\lambda) p}} \iint_{D}\left|\delta^{2-\lambda} \frac{\partial v}{\partial t}\right|^{p} d x .
$$

The last two inequalities together with (3.6) give, after integration with respect to time

$$
\left\|\left|\nabla^{2,1} f(s)\right|\right\|_{L_{p}(\Omega)}^{p} \leq C \frac{\theta^{p}(s)}{s^{(2-\lambda) p}}\|u\|_{L_{p}\left((0, T) ; B_{p}^{\lambda}(D)\right)}^{p} .
$$

Hence from (4.1), (4.2) and (4.3) we get that

$$
\begin{aligned}
\int_{0}^{\infty} & \left\|s^{\eta_{0}} f(s)\right\|_{W_{p}^{2,1}(\Omega)}^{p} \frac{d s}{s} \\
& \leq C\|u\|_{L_{p}\left((0, T) ; B_{p}^{\lambda}(D)\right.}^{p} \int_{0}^{\infty} s^{(2-\lambda+\varepsilon) p}\left(1+\frac{1}{s^{(1-\lambda) p}}+\frac{1}{s^{(2-\lambda) p}}\right) \theta^{p}(s) \frac{d s}{s} \\
& \leq C\|u\|_{L_{p}\left((0, T) ; B_{p}^{\lambda}(D)\right.}^{p} \int_{0}^{\frac{r}{4}} s^{(2-\lambda+\varepsilon) p}\left(1+\frac{1}{s^{(1-\lambda) p}}+\frac{1}{s^{(2-\lambda) p}}\right) \frac{d s}{s} \\
& =C\|u\|_{L_{p}\left((0, T) ; B_{p}^{\lambda}(D)\right.}^{p} \int_{0}^{\frac{r}{4}}\left(s^{(2-\lambda+\varepsilon) p}+s^{(1+\varepsilon) p}+s^{\varepsilon p}\right) \frac{d s}{s} .
\end{aligned}
$$

Since $\varepsilon>0$ we get that the first term in $(2.2)$ is finite.

In order to get an upper estimate for the second term in $(2.2)$, let us start by noticing that since $f^{\prime}(s)$ is the function $\frac{\partial v}{\partial y}\left(x^{\prime}, y+s ; t\right) \theta(s)+v\left(x^{\prime}, y+s ; t\right) \theta^{\prime}(s)$ we have that $f^{\prime \prime}(s)$ is the function 


$$
\frac{\partial^{2} v}{\partial y^{2}}\left(x^{\prime}, y+s ; t\right) \theta(s)+2 \frac{\partial v}{\partial y}\left(x^{\prime}, y+s ; t\right) \theta^{\prime}(s)+v\left(x^{\prime}, y+s ; t\right) \theta^{\prime \prime}(s)
$$

On the other hand, with the same arguments used to prove (4.1), (4.2) and (4.3) we obtain

$$
\begin{aligned}
\left\|v\left(x^{\prime}, y+s ; t\right) \theta^{\prime \prime}(s)\right\|_{L_{p}(\Omega)}^{p} \leq C\left|\theta^{\prime \prime}(s)\right|^{p}\|u\|_{L_{p}\left((0, T) ; B_{p}^{\lambda}(D)\right)}^{p} \\
\left\|\frac{\partial v}{\partial y}\left(x^{\prime}, y+s ; t\right) \theta^{\prime}(s)\right\|_{L_{p}(\Omega)}^{p} \leq C \frac{\left|\theta^{\prime}(s)\right|^{p}}{s^{(1-\lambda) p}}\|u\|_{L_{p}\left((0, T) ; B_{p}^{\lambda}(D)\right)}^{p} \\
\left\|\frac{\partial^{2} v}{\partial y^{2}}\left(x^{\prime}, y+s ; t\right) \theta(s)\right\|_{L_{p}(\Omega)}^{p} \leq C \frac{\theta(s)^{p}}{s^{(2-\lambda) p}}\|u\|_{L_{p}\left((0, T) ; B_{p}^{\lambda}(D)\right)}^{p}
\end{aligned}
$$

Hence, since $\theta, \theta^{\prime}$ and $\theta^{\prime \prime}$ are bounded and supported in $\left[-\frac{r}{4}, \frac{r}{4}\right]$ the second term on the left of $(2.2)$ is bounded by

$$
\begin{aligned}
& C\left(\int_{0}^{\frac{r}{4}} s^{\left(2-\frac{\lambda-\varepsilon}{2-\lambda+\varepsilon} \eta_{0}\right) p}\left(\frac{1}{s^{(2-\lambda) p}}+\frac{1}{s^{(1-\lambda) p}}+1\right) \frac{d s}{s}\right)\|u\|_{L_{p}\left((0, T) ; B_{p}^{\lambda}(D)\right)}^{p} \\
& \leq \widetilde{C}\left(\int_{0}^{\frac{r}{4}} s^{\eta_{0} p} \frac{d s}{s s^{(2-\lambda) p}}\right)\|u\|_{L_{p}\left((0, T) ; B_{p}^{\lambda}(D)\right)}^{p} \\
& =\widetilde{C}\left(\int_{0}^{\frac{r}{4}} \frac{d s}{s^{1-\varepsilon p}}\right)\|u\|_{L_{p}\left((0, T) ; B_{p}^{\lambda}(D)\right)}^{p} .
\end{aligned}
$$

ESTIMATES FOR $v=v_{\text {III }}$. In this case we shall use (2.b) in order to show that $v \in \mathbb{B}_{p}^{\lambda}(\Omega)$ which is better than the estimate obtained when $v$ is of type II. We shall check $(2.3)$ with $g(s)(x ; t)=v(x ; t+s) \omega(s)$ for $s>0$ and $\omega$ is a nonnegative $\mathscr{C}^{\infty}$ function supported in $\left(-r^{2}, r^{2}\right)$ with $\omega \equiv 1$ on $\left[-\frac{r^{2}}{2}, \frac{r^{2}}{2}\right], \alpha=\lambda$ and $\tau_{0}=1-\frac{\lambda}{2}$.

Let us start by estimating the first term on the left of (2.3) for the above described choice of $g, \alpha$ and $\tau_{0}$,

$$
\begin{aligned}
\|g(s)\|_{L_{p}(\Omega)}^{p} & =\iint_{\Omega}|v(x ; t+s) \omega(s)|^{p} d x d t \\
& =\omega^{p}(s) \iint_{\Omega}|v(x ; t+s)|^{p} d x d t \\
& =\omega^{p}(s) \int_{s}^{s+2 r^{2}}\left(\int_{D}|v(x ; t)|^{p} d x\right) d t \\
& \leq \omega^{p}(s) \iint_{\Omega}|v(x ; t)|^{p} d x d t .
\end{aligned}
$$

In the last inequality we have used that $r$ can be chosen so small that $3 r^{2}<T$. Hence the contribution of the $L_{p}(\Omega)$ norm of $g(s)$ to the Sobolev norm $W_{p}^{2,1}(\Omega)$ of $g(s)$ in the first term of $(2.3)$ is bounded by

$$
\left(\int_{0}^{\infty} s^{p\left(1-\frac{\lambda}{2}\right)} \omega^{p}(s) \frac{d s}{s}\right)\|v\|_{L_{p}(\Omega)}^{p} \leq C\left(\int_{0}^{r^{2}} s^{p\left(1-\frac{\lambda}{2}\right)} \frac{d s}{s}\right)\|u\|_{L_{p}\left((0, T) ; B_{p}^{\lambda}(D)\right)}^{p}
$$


which is finite.

Let us now get the bound of the term corresponding to the space gradient of $g(s)$ in the first term of $(2.3)$. For $i=1, \ldots, d$ we have that

$$
\begin{aligned}
\int_{0}^{\infty} s^{\tau_{0} p-1}\left\|\frac{\partial g}{\partial x_{i}}(s)\right\|_{L_{p}(\Omega)}^{p} d s & \left.\iint_{\left\{x \in D: d(x)>\frac{r}{2} ; 0<t<2 r^{2}\right\}}\left|\frac{\partial v}{\partial x_{i}}(x ; t+s)\right|^{p} d x d t\right) d s \\
& \left.\leq C \int_{0}^{r^{2}} s^{\left(1-\frac{\lambda}{2}\right) p-1} \int_{\left\{\int_{0}\right.} s^{\left(1-\frac{\lambda}{2}\right) p-1} \int_{\left\{x \in D: d(x)>\frac{r}{2} ; s<t<2 r^{2}+s\right\}}\left|\frac{\partial v}{\partial x_{i}}(x ; t)\right|^{p} d x d t\right) d s \\
& \leq C \int_{\left\{x \in D: d(x)>\frac{r}{2}\right\}} \int_{0}^{3 r^{2}}\left|\frac{\partial v}{\partial x_{i}}(x ; t)\right|^{p}\left(\int_{0}^{t} s^{\left(1-\frac{\lambda}{2}\right) p-1} d s\right) d t d x \\
& \leq C \int_{\left\{x \in D: d(x)>\frac{r}{2}\right\}} \int_{0}^{3 r^{2}}\left|t^{\left(1-\frac{\lambda}{2}\right)} \frac{\partial v}{\partial x_{i}}(x ; t)\right|^{p} d t d x \\
& \leq C \iint_{\Omega}\left|\delta^{2-\lambda}(x ; t) \frac{\partial v}{\partial x_{i}}(x ; t)\right|^{p} d x d t \\
& \leq C \iint_{\Omega}\left|\delta^{1-\lambda}(x ; t) \frac{\partial v}{\partial x_{i}}(x ; t)\right|^{p} d x d t
\end{aligned}
$$

where have used that for $(x ; t)$ in the support of $v, \delta(x ; t)$ is of the order $\sqrt{t}$ and that $r$ can be assumed to be less than 1 . Hence from (3.5) we get

$$
\int_{0}^{\infty} s^{\tau_{0} p-1}\left\|\frac{\partial g}{\partial x_{i}}(s)\right\|_{L_{p}(\Omega)}^{p} d s \leq C\|u\|_{L_{p}\left((0, T) ; B_{p}^{\lambda}(D)\right)}^{p} .
$$

In order to prove that the first term in (2.3) is finite we still have to deal with the second order space derivatives. Notice that to estimate

$$
\int_{0}^{\infty} s^{\tau_{0} p-1}\left\|\frac{\partial^{2} g}{\partial x_{j} \partial x_{i}}(s)\right\|_{L_{p}(\Omega)}^{p} d s
$$

in terms of $\iint_{\Omega}\left|\delta^{2-\lambda}(x ; t) \frac{\partial^{2} v}{\partial x_{j} \partial x_{i}}(x ; t)\right|^{p} d x d t$ we can proceed exactly as in (4.8) except for the last inequality there. The same is true for the time derivative. So that the desired result follows from (3.6).

Let us now accomplish the analysis of the second term in (2.3) for the current situation: $g(s)(x ; t)=v(x ; t+s) \omega(s), s>0, \alpha=\lambda$ and $\tau_{0}=1-\frac{\lambda}{2}$. Since for the derivative of $g$ with respect to $s$ we have

$$
g^{\prime}(s)(x ; t)=\frac{\partial v}{\partial t}(x ; t+s) \omega(s)+v(x ; t+s) \omega^{\prime}(s)
$$

and $\left(1-\frac{\alpha}{2-\alpha} \tau_{0}\right) p-1=\left(1-\frac{\lambda}{2}\right) p-1$, we can proceed exactly as in (4.7) and (4.8).

Estimates FOR $v=v_{\mathrm{IV}}^{i}$. As in the case of $v_{\mathrm{II}}^{i}$, from the invariance of the Laplacian we can assume that $0=p_{i} \in \partial D$ and that the coordinate system $x=$ 
$\left(x^{\prime}, y\right)$ with $x^{\prime} \in \mathbb{R}^{d-1}$ and $y \in \mathbb{R}$ is such that in the space ball $B(0, r), \partial D$ is the graph of the Lipschitz function $\varphi$.

In this case we apply (2.b) with $g(s)(x ; t)=v\left(x^{\prime}, y+s ; t+s^{2}\right) \theta(s), \theta$ as in the case $v=v_{\mathrm{II}}^{i}, \alpha=\lambda-\varepsilon$ and $\tau_{0}=2-\lambda+\varepsilon$ for $0<\varepsilon<\lambda$.

We have to prove that for this choice of $g, \alpha$ and $\tau_{0}$ the left hand side of (2.3) is finite. Let us start by noticing that, since $1-\frac{\alpha}{2-\alpha} \tau_{0}=1-\lambda+\varepsilon$ and $g^{\prime}(s)(x ; t)=$ $\frac{\partial v}{\partial y}\left(x^{\prime}, y+s ; t+s^{2}\right) \theta(s)+2 s \frac{\partial v}{\partial t}\left(x^{\prime}, y+s ; t+s^{2}\right) \theta(s)+v\left(x^{\prime}, y+s ; t+s^{2}\right) \theta^{\prime}(s)$, we have to show that each one of the following seven integrals is finite,

$$
\begin{aligned}
& A=\int_{0}^{\frac{r}{4}} s^{(2-\lambda+\varepsilon) p-1} \int_{0}^{2 r^{2}} \int_{B_{d-1}(0, r)} \int_{\varphi\left(x^{\prime}\right)}^{+\infty}\left|v\left(x^{\prime}, y+s ; t+s^{2}\right)\right|^{p} d y d x^{\prime} d t d s \\
& B=\int_{0}^{\frac{r}{4}} s^{(2-\lambda+\varepsilon) p-1} \int_{0}^{2 r^{2}} \int_{B_{d-1}(0, r)} \int_{\varphi\left(x^{\prime}\right)}^{+\infty}\left|\frac{\partial v}{\partial x_{i}}\left(x^{\prime}, y+s ; t+s^{2}\right)\right|^{p} d y d x^{\prime} d t d s
\end{aligned}
$$

for $i=1, \ldots, d$ and $x_{d}=y$;

$$
\begin{aligned}
& C=\int_{0}^{\frac{r}{4}} s^{(2-\lambda+\varepsilon) p-1} \int_{0}^{2 r^{2}} \int_{B_{d-1}(0, r)} \int_{\varphi\left(x^{\prime}\right)}^{+\infty}\left|\frac{\partial^{2} v}{\partial x_{j} \partial x_{i}}\left(x^{\prime}, y+s ; t+s^{2}\right)\right|^{p} d y d x^{\prime} d t d s ; \\
& D=\int_{0}^{\frac{r}{4}} s^{(2-\lambda+\varepsilon) p-1} \int_{0}^{2 r^{2}} \int_{B_{d-1}(0, r)} \int_{\varphi\left(x^{\prime}\right)}^{+\infty}\left|\frac{\partial v}{\partial t}\left(x^{\prime}, y+s ; t+s^{2}\right)\right|^{p} d y d x^{\prime} d t d s ; \\
& E=\int_{0}^{\frac{r}{4}} s^{(1-\lambda+\varepsilon) p-1} \int_{0}^{2 r^{2}} \int_{B_{d-1}(0, r)} \int_{\varphi\left(x^{\prime}\right)}^{+\infty}\left|\frac{\partial v}{\partial y}\left(x^{\prime}, y+s ; t+s^{2}\right)\right|^{p} d y d x^{\prime} d t d s ; \\
& F=\int_{0}^{\frac{r}{4}} s^{p} s^{(1-\lambda+\varepsilon) p-1} \int_{0}^{2 r^{2}} \int_{B_{d-1}(0, r)} \int_{\varphi\left(x^{\prime}\right)}^{+\infty}\left|\frac{\partial v}{\partial t}\left(x^{\prime}, y+s ; t+s^{2}\right)\right|^{p} d y d x^{\prime} d t d s ; \\
& G=\int_{0}^{\frac{r}{4}} s^{(1-\lambda+\varepsilon) p-1} \int_{0}^{2 r^{2}} \int_{B_{d-1}(0, r)} \int_{\varphi\left(x^{\prime}\right)}^{+\infty}\left|v\left(x^{\prime}, y+s ; t+s^{2}\right)\right|^{p} d y d x^{\prime} d t d s .
\end{aligned}
$$

Since $A \leq G, D=F$ and $B$ and $E$ are similar, we only have to show that $G, E$, $C$ and $D$ are finite.

Bound for $G$. Changing variables in space variable $y$ and in time variable $t$ we have that

$$
\begin{aligned}
G & =\int_{0}^{\frac{r}{4}} s^{(1-\lambda+\varepsilon) p-1} \int_{s^{2}}^{2 r^{2}+s^{2}} \int_{B_{d-1}(0, r)} \int_{\varphi\left(x^{\prime}\right)+s}^{+\infty}\left|v\left(x^{\prime}, y ; t\right)\right|^{p} d y d x^{\prime} d t d s \\
& \leq \int_{0}^{\frac{r}{4}} s^{(1-\lambda+\varepsilon) p-1} \int_{0}^{3 r^{2}} \int_{B_{d-1}(0, r)} \int_{\varphi\left(x^{\prime}\right)}^{+\infty}\left|v\left(x^{\prime}, y ; t\right)\right|^{p} d y d x^{\prime} d t d s \\
& \leq \int_{0}^{\frac{r}{4}} s^{(1-\lambda+\varepsilon) p-1} d s \iint_{\Omega}|v|^{p} d x d t \\
& \leq C\|u\|_{L_{p}(\Omega)}^{p}
\end{aligned}
$$

Bound for $E$. Since

$$
E=\int_{0}^{\frac{r}{4}} s^{\varepsilon p-1} \int_{0}^{2 r^{2}} \int_{B_{d-1}(0, r)} \int_{\varphi\left(x^{\prime}\right)}^{\infty} s^{(1-\lambda) p}\left|\frac{\partial v}{\partial y}\left(x^{\prime}, y+s ; t+s^{2}\right)\right|^{p} d y d x^{\prime} d t d s,
$$


and in the domain of integration the inequality $\delta\left(x^{\prime}, y+s ; t+s^{2}\right) \geq c s$ holds for some constant $c$, after changing variables, we obtain from (3.5) that

$$
E \leq c\left(\int_{0}^{\frac{r}{4}} s^{\varepsilon p-1} d s\right)\left(\iint_{\Omega}\left|\delta^{1-\lambda} \frac{\partial v}{\partial y}\right|^{p} d x d t\right) \leq C\|u\|_{L_{p}\left((0, T) ; B_{p}^{\lambda}(D)\right)}^{p} .
$$

Bound for $C$ and $D$. Since $C$ and $D$ both can be written in the form

$$
\int_{0}^{\frac{r}{4}} s^{\varepsilon p-1} \int_{0}^{2 r^{2}} \int_{B_{d-1}(0, r)} \int_{\varphi\left(x^{\prime}\right)}^{\infty} s^{(2-\lambda) p}\left|V\left(x^{\prime}, y+s ; t+s^{2}\right)\right|^{p} d y d x^{\prime} d t d s
$$

with $V=\frac{\partial^{2} v}{\partial x_{j} \partial x_{i}}$ for $C$ and $V=\frac{\partial v}{\partial t}$ for $D$, and $\delta\left(x^{\prime}, y+s ; t+s^{2}\right) \geq c s$, we can now use (3.6) to obtain that they are bounded by a constant times $\|u\|_{L_{p}\left((0, T) ; B_{p}^{\lambda}(D)\right)}^{p}$.

\section{REFERENCES}

[AGI] Hugo Aimar, Ivana Gómez, and Bibiana Iaffei, Parabolic mean values and maximal estimates for gradients of temperatures, J. Funct. Anal. 255 (2008), no. 8, 1939-1956.

[BL] Jöran Bergh and Jörgen Löfström, Interpolation spaces. An introduction, SpringerVerlag, Berlin, 1976, Grundlehren der Mathematischen Wissenschaften, No. 223.

[BS] Colin Bennett and Robert Sharpley, Interpolation of operators, Pure and Applied Mathematics, vol. 129, Academic Press Inc., Boston, MA, 1988.

[DD] Stephan Dahlke and Ronald DeVore, Besov regularity for elliptic boundary value problems, Comm. Partial Differential Equations 22 (1997), no. 1-2, 1-16.

[HS] Yong Sheng Han and Eric Sawyer, Littlewood-Paley theory on spaces of homogeneous type and the classical function spaces, Mem. Amer. Math. Soc. 110 (1994), no. 530, vi+126.

[JK] David Jerison and Carlos Kenig, The inhomogeneous Dirichlet problem in Lipschitz domains, J. Funct. Anal. 130 (1995), no. 1, 161-219.

[JM] Tünde Jakab and Marius Mitrea, Parabolic initial boundary value problems in nonsmooth cylinders with data in anisotropic Besov spaces, Math. Res. Lett. 13 (2006), no. 5-6, 825831.

[Pe] Jaak Peetre, New thoughts on Besov spaces, Duke University Mathematics Series, vol. 1, Mathematics Department, Duke University, Durham, N.C., 1976.

Instituto de Matemática Aplicada del Litoral (CONICET-UNL), GüEmes 3450 - S3000GLN Argentina

E-mail address: haimar@santafe-conicet.gov.ar

E-mail address: ivanagomez@santafe-conicet.gov.ar

E-mail address: biaffei@santafe-conicet.gov.ar 